\title{
EFECTO DEL USO DE TUTORES Y APLICACIÓN DE BIOFERTILIZANTES EN EL CRECIMIENTO Y DESARROLLO DE Plukenetia volubilis L. "SACHA INCHI"
}

\author{
Percy DIAZ1 ${ }^{1}$ Cheryl TELLO ${ }^{2}$, Luis AREVALO $^{1}$ \\ 1 Instituto de Investigaciones de la Amazonía Peruana (IIAP). Programa de Investigación en Manejo Integral del Bosque y \\ Servicios Ambientales (PROBOSQUES). San Martín, Perú. E-mail: pdiaz@iiap.org.pe; larevalo@iiap.org.pe \\ 2 Investigador en manejo de cultivos tropicales, elaboración y aplicación de abonos orgánicos.
}

\section{RESUMEN}

Se evaluó el efecto de dos tutores y cinco tipos de biofertilizantes en el crecimiento y desarrollo de Plukenetia volubilis L. sacha inchi en un suelo de baja fertilidad. La plantación se estableció utilizando el diseño de bloques completos al azar en parcelas divididas, siendo las parcelas principales dos sistemas de tutor y las sub parcelas cinco tipos de biofertilizantes. Los cinco biofertilizantes fueron: Biol, Biol mas frutos de Solanum torvum, Biol más hojas de Carica papaya, Biol más hojas de Cymbopogon citratus y Biol más hojas de Chondrodendron tomentosum, más un testigo (SB) por cada sistema de tutor, teniendo un total de doce tratamientos y tres bloques. Las dosis de aplicación fueron de 15 L.ha $^{-1}$, divididas en aplicaciones quincenales a razón de $1.5 \mathrm{~L} \mathrm{ha}^{-1}$ en campo definitivo, las evaluaciones fueron realizadas cada quince días durante 5 meses. Los resultados indican que con los tratamientos T4 y T8 se redujeron los días para la fructificación, lográndose el mayor número de frutos con los tratamientos T4, T9 y T10, mejorando la productividad de plantas de $P$. volubilis $\mathrm{L}$.

PALABRAS CLAVE: Plukenetia volubilis L., biofertilizantes, productividad

\section{EFFECT OF THE USE AND APPLICATION OF GUARDIANS BIOFERTILIZERS ON GROWTH AND DEVELOPMENT OF Plukenetia volubilis L. "SACHA INCHI"}

\section{ABSTRACT}

The objective was to evaluate the effect of two tutors and five types of biofertilizers on growth and development of Plukenetia volubilis L. in a soil of low fertility. The plantation was established using design randomized complete block split plot, with the two sets of main plots and sub plots tutor five types of biofertilizers. Biofertilizer five were: Biol, Biol more fruits of Solanum torvum, Biol more Carica papaya leaves, Biol more Cymbopogon citratus sheets of and Biol more Chondrodendron tomentosum sheets, and a control (SB) for each tutor system, having a total twelve treatments and three blocks. The application rate was 15 l.ha ${ }^{-1}$, divided into fortnightly applications by 1.51. ha $^{-1}$ final field evaluations were performed every two weeks for 5 months. The results indicate that the T4 and T8 treatments reduced fruit set days and the highest number of fruits per plant was achieved with $\mathrm{T} 4, \mathrm{~T} 9$ and $\mathrm{T} 10$ treatments, improving the productivity of plants of $P$. volubilis $\mathrm{L}$.

KEYWORDS: Plukenetia volubilis L., bio-fertilizers, productivity 


\section{INTRODUCCIÓN}

El Sacha Inchi (Plukenetia volubilis L), es una planta arbustiva que pertenece a la familia Euphorbiaceae (Mcbride, 1951), también es conocido como Maní Silvestre o Inca Inchi. Es una planta silvestre de la Amazonía peruana, encontrándose en las regiones de San Martín, Ucayali, Loreto, Cusco y Madre de Dios (Peña et al., 2008; Márquez et al., 2007), tiene un gran potencial agroindustrial, por la cantidad de proteínas que contiene $(23 \%)$ y por su contenido de aceites $(49,9 \%)$ (Castillo et al. 2010), entre los que destacan los aceites ricos en omega 3, 6 y 9 (Merino et al. 2008 y Gorriti et al. 2010). Es una planta que por su rusticidad se adapta a diferentes tipos de suelo (Arévalo, 1996).

Actualmente se presenta en el mundo una tendencia a la producción y consumo de alimentos obtenidos de manera limpia, es decir, sin el uso de pesticidas y fertilizantes químicos. La tendencia a la producción orgánica de alimentos es una alternativa que beneficia tanto a productores como a consumidores (Alcívar et al, 2012)

El uso del Biol, se plantea como una alternativa de abono orgánico, orientado a mejorar la productividad y calidad de los cultivos. Los "bioles" son un tipo de biofertilizante líquido (obtenido de fermentación anaeróbica), ricos en micronutrientes, fitohormonas y microorganismos benéficos. Se utilizan como abono, estimuladores del crecimiento vegetal e inductores a respuestas fisiológicas como floración y fructificación; recientemente se ha comprobado su efecto como inhibidor del crecimiento de hongos fito patógenos (Álvarez et al, 2011), con eficiencia en sectores donde hay limitaciones causadas por el estrés fisiológico (Bejarano et al, 2004).

Por las razones expuestas, el objetivo de la investigación fue evaluar la aplicación de cinco biofertilizantes en el crecimiento y desarrollo del sacha inchi.

\section{MATERIALES Y MÉTODOS}

\section{Ubicación del campo experimental}

El trabajo de investigación se llevó a cabo en el Centro Poblado de Patria, a 207.35 Km. de la ciudad de Cusco, (carretera Cusco - Paucartambo Pillcopata), con una duración de 8 meses; ubicado a $12^{\circ} 53^{\prime}$ Latitud Sur, $71^{\circ} 25^{\prime}$ Longitud Oeste y a 659 msnm.

Durante el experimento la temperatura media fue de $21,6^{\circ} \mathrm{C}$, la precipitación anual de $3219 \mathrm{~mm}$ y la humedad relativa promedio de $89,3 \%$. Se tomó una muestra de suelo para determinar sus condiciones nutricionales, siendo etiquetada y llevada al laboratorio de suelos de la Universidad Nacional Agraria la Molina, el resultado del análisis se presenta en la Tabla 1.

Se empleó un diseño de bloques completos al azar (DBCA), en parcelas divididas; teniendo como parcelas principales dos sistemas de tutor: 1. Tutor Vivo y 2. Espalderas; en sub parcelas cinco tipos de biofertilizantes descritos en la tabla 2 , más un testigo por cada sistema de tutor, con un total de 12 tratamientos y tres bloques. El análisis de los resultados se realizó con el software estadístico SAS (Statistical Analysis System, 2004) y para la comparación de medias se utilizó el análisis de varianza y la prueba de Duncan (Gómez \& Gómez, 1984).

Se evaluaron la altura de la planta, el número de flores por planta y el número de frutos por planta. Para evaluar la altura de la planta $(\mathrm{cm})$, se inició una revisión a los 8 días después del trasplante, realizando una medida por semana durante 6 semanas. Se registraron los días transcurridos después del trasplante hasta que las plantas alcanzaron 50\% de emisión. Para evaluar el número de flores por planta, se contabilizó el número de racimos florales formados hasta la aparición de los primeros frutos. Por último, se para evaluar el número de frutos por planta, se registró la cantidad de frutos listos para la cosecha.

\section{Preparación de los Biofertilizantes}

Para obtener los biofertilizantes, se siguió el procedimiento descrito por Soria et al 2001, quien indica que los insumos deben pasar por un periodo de fermentación anaeróbica, por espacio de 45 días en un digestor artesanal.

\section{Construcción de los digestores}

Se construyeron cinco digestores tipo Batch (Seguel et al, 2003), utilizando para ello 5 timbos de 100 L., cuya tapa tenía un agujero de $1 / 2$ pulgada, insertada con una manguera transparente de $1 / 2$ pulgada y de dos metros de largo, en el extremo externo de cada manguera se introdujo una botella descartable de $1 \mathrm{~L}$. con agua no clorada hasta la $3 / 4$ partes, como una trampa de gas, facilitando la salida del gas y no permitiendo el ingreso de oxígeno para una buena fermentación.

\section{Preparación de los biofertilizantes}

En cada digestor se colocaron $10 \mathrm{~kg}$ de estiércol de vacuno, $2 \mathrm{~kg}$ de rumen de ganado vacuno, $2 \mathrm{~kg}$ de 
ceniza, $0,250 \mathrm{~kg}$ de cáscara de huevo molido, 0,250 $\mathrm{kg}$ de azúcar, $250 \mathrm{ml}$ de leche y fermento de maíz, como mezcla base. Para diferenciar los tratamientos en cada digestor se colocó en cada uno un tipo de planta, $1 \mathrm{~kg}$ de hojas de "papaya" (Carica papaya), $1 \mathrm{~kg}$ de hojas de "Hierba luisa" (Cymbopogon citratus), $1 \mathrm{~kg}$ de "curare" (Chondrodendron tomentosum), por último, $1 \mathrm{~kg}$ de frutos de "teta de vaca" (Solanum mammosum), tal como figura en la Tabla 2. Se añadió agua no clorada a cada digestor, dejando $20 \mathrm{~cm}$ de espacio entre el nivel del agua y la tapa (cámara de gases). Todos los insumos se mezclaron de forma homogénea y se cerró herméticamente para facilitar la fermentación. Todo este proceso fue desarrollado bajo las metodologías combinadas de Criollo et al (2011) y Litterick \& Wood (2009), en las instalaciones del Instituto Nacional de Innovación Agraria (INIA), sede Pillcopata, Cusco.

La cosecha de los biofertilizantes se realizó a los 45 días después de su preparación, utilizando un tamiz para evitar el paso de partículas que puedan obstruir el pulverizador. El líquido fue guardado en recipientes de 20 L., debidamente rotuladas, para su aplicación en campo y posterior análisis, se extrajo un litro de cada biofertilizante, debidamente rotulado y llevado al laboratorio de suelos de la Universidad Nacional Agraria La Molina (Tabla 3).

\section{Siembra en cama almaciguera y parcela experimental}

Se instaló una cama almaciguera de $1 \mathrm{~m}$. de ancho por $2 \mathrm{~m}$. de largo, construida con materiales de la zona. Las semillas utilizadas fueron de plantas seleccionadas del ecotipo pinto recodo, sembradas en la cuidad de Pucallpa - Perú. El sustrato fue de estiércol de vacuno descompuesto, tierra agrícola y ceniza de cascarilla de arroz en una proporción de 3:1:1. Las bolsas almacigueras fueron de 5" $x 7$ ". Las semillas de Sacha inchi se trataron con $250 \mathrm{~g}$ de ceniza de cascarilla de arroz en medio litro de agua por 5 horas.
La parcela experimental fue instalada mediante el rozo, tumba, sin quema, en un área de $2500 \mathrm{~m}^{2}$. Como tutor vivo se utilizó la Erythrina sp, de $2 \mathrm{~m}$ de largo, y de $8 \mathrm{~cm}$ de diámetro, siendo sembrados a 30 $\mathrm{cm}$ de profundidad y distanciados a 3 x $3 \mathrm{~m}$ (1 111 tutores ha $\left.{ }^{-1}\right)$. En el caso de la espaldera se utilizaron postes de 2,5 $\mathrm{m}$ de largo por 3" de en distanciamiento $3 \mathrm{~m} \times 6 \mathrm{~m}$. Se templaron dos hileras de alambre galvanizado $\mathrm{N}^{\mathrm{o}} 16$ a $0.80 \mathrm{~m}$ y $1.80 \mathrm{~m}$ del suelo.

El trasplante fue a los 15 días después de la emergencia, en hoyos de $15 \times 15 \times 25 \mathrm{~cm}$ a $15 \mathrm{~cm}$ del tutor vivo y a distancia de $3 \times 3 \mathrm{~m}$ (1 111 plantas. ha $\left.{ }^{1}\right)$.

\section{Aplicación de los biofertlizantes}

La dosis de aplicación para los diferentes biofertilizantes fue de 15 L.ha $^{-1}$. Esta dosis fue fraccionadaen 10 aplicaciones, realizándose cada 15 días a partir del octavo día después del trasplante, usando una pulverizadora de $15 \mathrm{~L}$. con 1,5 L. de biofertilizantes más 13,5 L. de agua.

\section{Manejo del cultivo}

Se realizaron podas de formación a los 30 días después del trasplante y despunte (corte de la guía) dependiendo del tipo de tutor; así en el "tutor vivo" (TV) la poda se realizó cuando el tallo guía alcanzó la primera ramificación de la horqueta y en la "espaldera" (E) a $20 \mathrm{~cm}$ de distancia del primer alambre, esto permitió el crecimiento de ramas secundarias que se acomodaron en el primer alambre de la espaldera y en las ramas del tutor vivo.

\section{Control fitosanitario}

Cada 15 días se aplicó extracto de ajo (Allium sativum L.), para reducir el daño por enfermedades y extracto de ají (Capsicum sp L.), para el ataque de insectos cortadores. Se prepararon bajo la metodología descrita por De Marcano (2005) y Castillo-Sánchez et al (2012), colocando 100 g. en medio litro de agua, macerando por 24 horas y diluidos en $15 \mathrm{~L}$. de agua antes de cada aplicación. 
Tabla 1. Análisis físico-químico del suelo.

\begin{tabular}{|c|c|c|c|c|}
\hline \multirow{2}{*}{ Muestra de suelo } & \multicolumn{2}{|c|}{ Resultado } & \multirow{2}{*}{ Interpretación } & \multirow{2}{*}{ Método } \\
\hline & Unidades & Kg./ha. & & \\
\hline \multicolumn{5}{|l|}{ PARÁMETROS } \\
\hline Textura & & & Frc. Arenoso & Bouyucos \\
\hline Arena & $56 \%$ & & & \\
\hline Arcilla & $12 \%$ & & & \\
\hline Limo & $32 \%$ & & & \\
\hline C. E. & $0,09 \mathrm{dS} \cdot \mathrm{m}^{-1}$ & & No salino & Conductimétro \\
\hline pH & 3,99 & & Fuertemente ácido & Potenciómetro \\
\hline Materia Orgánica & $2,3 \%$ & & Medio & Walkley Black \\
\hline Fósforo disponible & 4,4 & & Bajo & Olsen Mod. \\
\hline Potasio Interc. & $0,13 \mathrm{cmol} . \mathrm{kg}^{-1}$ & 21,7 & Bajo & Ác. Ascórbico \\
\hline Calcio. & 1,28 cmol. $\mathrm{kg}^{-1}$. & 140,0 & Bajo & Tetra. Borato \\
\hline Magnesio & $0,25 \mathrm{cmol}^{\mathrm{kg}}{ }^{-1}$ & & Bajo & Titulación EDTA \\
\hline Aluminio & $4,0 \mathrm{cmol} \cdot \mathrm{kg}^{-1}$ & & Posible toxicidad & \\
\hline CIC & $12,8{\mathrm{cmol} . \mathrm{kg}^{-1}}^{-1}$ & & Medio & \\
\hline
\end{tabular}

Fuente: Laboratorio de suelos de la Universidad Nacional Agraria La Molina - Lima (2007).

Tabla 2. Tratamientos en estudio.

\begin{tabular}{|c|c|c|}
\hline Trat. & $\begin{array}{l}\text { Parcela Principal: } \\
\text { Sistema de tutor }\end{array}$ & $\begin{array}{l}\text { Sub parcelas: } \\
\text { Biofertilizantes }\end{array}$ \\
\hline T1 & Tutor vivo (TV) & $\mathbf{B}+\mathbf{F T V}=$ Biol con frutos de "Teta de vaca" (Solanum mammosum) \\
\hline T2 & & $\mathbf{B}+\mathbf{H P}=$ Biol con hojas de "Papaya" (Carica papaya) \\
\hline T3 & & B $+\mathbf{H H L}=$ Biol con hojas de "Hierba luisa" (Cymbopogon citratus) \\
\hline T4 & & $\mathbf{B}+\mathbf{H C}=$ Biol con hojas de "Curare" (Chondrodendron tomentosum) \\
\hline T5 & & $\mathbf{B}=$ Biol \\
\hline T6 & & SB $=$ Testigo (sin biol) \\
\hline T7 & Espalderas (E) & $\mathbf{B}+\mathbf{F T V}=$ Biol con frutos de "Teta de vaca" (Solanum mammosum) \\
\hline T8 & & $\mathbf{B}+\mathbf{H P}=$ Biol con hojas de "Papaya" (Carica papaya) \\
\hline T9 & & B $+\mathbf{H H L}=$ Biol con hojas de "Hierba luisa" (Cymbopogon citratus) \\
\hline T10 & & $\mathbf{B}+\mathbf{H C}=$ Biol con hojas de "Curare" (Chondrodendron tomentosum) \\
\hline T11 & & $\mathbf{B}=$ Biol \\
\hline T12 & & SB $=$ Testigo (sin biol) \\
\hline
\end{tabular}


Tabla 3. Resultados del Análisis químico de los Bioles en estudio.

\begin{tabular}{|c|c|c|c|c|c|c|}
\hline \multicolumn{7}{|c|}{ Resultados } \\
\hline Análisis & Unid. & B & $\mathrm{B}+\mathrm{FTV}$ & $B+H P$ & $\mathrm{~B}+\mathrm{HHL}$ & $B+H C$ \\
\hline Sólidos en suspensión & g..$^{-1}$ & 18,56 & 15,58 & 16,72 & 15,70 & 15,06 \\
\hline M.0. en solución & g..$^{-1}$ & 6,86 & 5,94 & 6,44 & 5,84 & 5,46 \\
\hline pH & & 7,5 & 7,5 & 7,3 & 7,4 & 7,2 \\
\hline Conductividad Elect. & $\mathrm{dS} . \mathrm{m}^{-1}$ & 9,87 & 9,62 & 10,18 & 9,39 & 9,50 \\
\hline Nitrógeno Total & $\mathrm{mg} \cdot \mathrm{I}^{-1}$ & 521,0 & 544,0 & 532,0 & 420,0 & 448,0 \\
\hline Fósforo Total & $\left.m g\right|^{-1}$ & 182,0 & 68,0 & 78,0 & 108,0 & 104,0 \\
\hline Potasio Total & $\left.m g\right|^{-1}$ & 3280,0 & 3040,0 & 3160,0 & 3240,0 & 3520,0 \\
\hline Calcio Total & $\mathrm{mgl}^{-1}$ & 185,2 & 166,4 & 173,2 & 149,2 & 170,8 \\
\hline Magnesio Total & $\left.m g\right|^{-1}$ & 159,0 & 164,0 & 160,0 & 152,0 & 180,0 \\
\hline Sodio Total & $\left.m g\right|^{-1}$ & 608,0 & 296,0 & 296,0 & 240,0 & 280,0 \\
\hline Hierro Total & $\mathrm{mgl}^{-1}$ & 20,3 & 21,4 & 18,0 & 14,1 & 11,6 \\
\hline Cobre Total & $\left.m g\right|^{-1}$ & 1,60 & 0,24 & 0,40 & 0,52 & 0,28 \\
\hline Zinc Total & $\mathrm{mgl}^{-1}$ & 0,84 & 0,44 & 0,48 & 0,40 & 0,40 \\
\hline Manganeso Total & $m g l^{-1}$ & 7,84 & 5,76 & 5,64 & 4,88 & 5,68 \\
\hline Boro Total & $m g l^{-1}$ & 1,3 & 1,2 & 0,9 & 1,0 & 1,5 \\
\hline
\end{tabular}

Fuente: Laboratorio de suelos de la Universidad Nacional Agraria La Molina - Lima (2007).

\section{RESULTADOS Y DISCUSIONES}

El análisis químico de los cinco biofertilizantes muestra diferencias entre ellas, pero en común todos los biofertilizantes son fuertemente salinos, siendo el biofertilizante $\mathrm{B}+\mathrm{HP}$, el de mayor C.E., esto podría deberse a que el estiércol líquido de por si tiene alta conductividad eléctrica (CE), lo cual es corroborado por Soria et al (2001),que encontró valores cercanos a $15 \mathrm{dS} \cdot \mathrm{m}^{-1}$, al usar estiércol de cerdo, esto se soluciona al hacer diluciones del estiércol líquido en agua, como lo mencionan Capulin-Grande et al.(2007).

Con respecto al biofertilizante $\mathrm{B}$, presentó altos contenidos de sólidos en suspensión $\left(18,56 \mathrm{gl}^{-1}\right)$, materia orgánica en solución $\left(6,86 \mathrm{gl}^{-1}\right)$, fósforo $(182$ $\left.\mathrm{mgl} \mathrm{l}^{-1}\right)$, Calcio $\left(185,2 \mathrm{mgl}^{-1}\right)$, Sodio $\left(608 \mathrm{mg}^{-1}\right)$ cobre $\left(1,60 \mathrm{mgl}^{-1}\right)$, Zinc $\left(0,84 \mathrm{mgl}^{-1}\right)$ y Manganeso $(7,84$ $\left.\mathrm{mgl} \mathrm{l}^{-1}\right)$, mientras que el biofertilizante B+FTV tuvo altos contenidos de Nitrógeno (544 $\mathrm{mgl}^{-1}$ ) y Hierro $\left(21,4 \mathrm{mgl}^{-1}\right)$, en el biofertilizante $\mathrm{B}+\mathrm{HC}$ se encontraron altos contenidos de potasio (3 520 $\left.\mathrm{mgl} \mathrm{l}^{-1}\right)$, magnesio $\left(180 \mathrm{mgl}^{-1}\right)$ y Boro $\left(1,3 \mathrm{mgl}^{-1}\right)$; todos los biofertilizantes tienen $\mathrm{pH}$ ligeramente alcalinos; los resultados de los análisis corroboran lo mencionado por Sánchez (2009), que indica que los desechos de las fincas, como el estiércol bovino, mezclado con residuos vegetales y algunos aditivos, constituyen una alternativa para la producción de los abonos orgánicos líquidos y sólidos, puesto que son una fuente rica en nutrientes (Tabla 3 ).

Generalmente los bioles son utilizados con el propósito de mejorar la nutrición de los cultivos y también como estimuladores del crecimiento, floración y fructificación de los cultivos con resultados favorables (Basantes, 2010).

La utilización de los biofertiliantes es una alternativa viable y económica, ya que se aprovecha un desecho que propicia el reciclamiento de nutrimentos en la producción agrícola. La baja concentración de algunos elementos en el estiércol líquido se puede corregir adicionando fertilizantes (Rodríguez etal., 2007).

\section{Efecto de los biofertilizantes líquidos en el crecimiento y desarrollo de Sacha Inchi (Plukenetia volubilis $\mathrm{L}$.)}

Las plantas biofertilizadas tuvieron mejor crecimiento y desarrollo, esta respuesta se debe a que los biofertilizantes líquidos son una fuente 
orgánica de nutrientes que permite promover actividades fisiológicas y estimular el desarrollo de las plantas (Gomero \& Velásquez, 1999). Además, de acuerdo a los resultados se puede deducir que los nutrientes de los biofertilizantes fueron asimilados rápidamente por las plantas, debido a la aplicación foliar, siendo esto corroborado por Perdomo \& Hampton (1970), quienes afirman que los nutrientes que pueden ser aplicados eficientemente por aspersiones foliares son: nitrógeno, fósforo, potasio, calcio, magnesio, azufre, hierro, manganeso, zinc, molibdeno. Además, señalan que los macro elementos pueden ser aplicados en aspersiones, únicamente como suplemento nutricional a los cultivos durante los períodos críticos del crecimiento.

Los sistemas de tutor vivo y espaldera, según el análisis de varianza, son significativos (tabla 4) para el número de flores y frutos por planta, ya que en espaldera la planta de Sacha inchi tiene más facilidad de expansión que en tutor vivo, estos resultados son similares con los encontrados en vainilla (Vanilla planifolia Andrews) por Elorza et al (2007), al estudiar el contenido de clorofila, estableciendo mayor contenido en plantas bajo los sistemas de espaldera, seguido del tutor Erythrina ya que al existir mayor entrada de luz mayor asimilación del nitrógeno para la producción de clorofila y procesos fotosintéticos y reproductivos.

Los biofertilizantes aplicados muestran diferencias significativas (tabla 4) en las diversas variables estudiadas ( $>>0,001)$, Gómez \& Gómez (1984), corroborado con la prueba de rangos múltiple de Duncan $(\mathrm{p}<0,05)$. Las mejor altura de planta se han logrado al aplicar el tratamiento T2 y T8 (en espaldera y tutor vivo) con $29,7 \mathrm{~cm}$ y $27,9 \mathrm{~cm}$. Estos resultados corroboran lo mencionado por Dávila (2008), que obtuvo las mayores altura a dosis de biol al 100\%, en el cultivo de espinaca (Spinacia oleracea L.).

En lo referente a la emisión de guía, la prueba de Duncan $(\mathrm{p}<0,05)$, nos muestra que el tratamiento T8 fue el mejor, logrando promedios de 38 días después del trasplante, este resultado está dentro del rango mencionado por Manco (2006), que señala que está entre los 20 y 41 días después del trasplante, por lo que se deduce que este tratamiento tiene poder estimulante del crecimiento en las plantas de Sacha inchi, por la presencia de nitrógeno de $532 \mathrm{mg}^{-1}$ que estimula el crecimiento de tallos y hojas (Kovacik et ál. 2007).

En cuanto a la floración, con el tratamiento $\mathrm{T} 1$ en tutor vivo fue a los 79 días, al igual que T4. En espaldera fue a los 77 días, después del trasplante con el tratamiento $\mathrm{T} 7$; mientras que para los tratamientos T8, T9 y T10, se logró a los 74 días, siendo estadísticamente iguales. Estos resultados indican que los tratamientos de biofertilizantes acortaron el periodo de floración, de acuerdo con los resultados de Manco (2006), quien registró el inicio de floración entre los 86 y 139 días después del trasplante. Estos resultados están influenciados por la presencia de diferentes elementos como el fósforo, que estimula la floración, así como la presencia de microelementos como el boro y el cobre, que favorecen la formación de flores (Xiangwen et ál. 2008; Marschner 1995).

En el caso del número de flores por planta, los resultados son notorios en el sistema de Espaldera, con un promedio de 55 flores por planta con el tratamiento $\mathrm{T} 7$, mientras con tutor vivo el promedio fue de 34 flores por planta con el tratamiento $\mathrm{T} 4$.

En lo referente a la fructificación de las plantas de Sacha inchi, las mejores respuestas se obtuvieron con el tratamiento T4 y T8 para tutor vivo, a los 115 días y para espaldera, a los 109 días, estando muy cerca de los resultados obtenidos por Manco (2006), por debajo del rango de 119 y 182 días después del trasplante.

Con los tratamientos T4 se tuvo mayor respuesta en el número de frutos por planta, siendo de 40 frutos en tutor vivo y de 65 y 66 frutos en espaldera con los tratamientos T9 y T10 respectivamente. Se puede notar la influencia significativa de los sistemas de tutorado en el número de frutos, como en el caso del número de flores por planta explicado anteriormente.

El Sacha inchi es una planta agronómicamente rústica, de poca exigencia nutricional; se adapta a tipos de suelo de distinta textura: arcillosos, francos y franco-arenosos, con $\mathrm{pH}$ entre 4,5 y más de 6,5 (Castro, 2007), en esta investigación, en base al análisis de suelo realizado en el área del experimento, se puede decir que soporta un $\mathrm{pH}$ de 3,99 , lo que se traduce en suelos fuertemente ácidos.

Los resultados obtenidos demuestran que las aplicaciones de biofertilizantes a dosis de 15 L.ha ${ }^{-1}$ estimulan el crecimiento de las plantas y mejoran la calidad de los frutos.

Así mismo, observando los resultados obtenidos en el crecimiento y desarrollo del Sacha inchi y los resultados de los análisis de los biofertilizantes, se corrobora los descrito por Bonilla (2000), que menciona como las plantas por si mismas tienen una capacidad limitada para la absorción selectiva de aquellos minerales que no son necesarios para su crecimiento.

Estas respuestas en el crecimiento y desarrollo del Sacha inchi corresponden a la fracción liquida, 
rica en nutrientes, con numerosos efectos positivos en los cultivos cuando son aplicados al follaje de las plantas o al suelo (Litterick \& Wood, 2009). La escasez de nitrógeno, fósforo, potasio y agua se convierte en una limitante para el crecimiento, desarrollo y rendimiento económico de los cultivos (Parry et al. 2005). El limitado suministro de N, P y K disminuye la tasa de división celular, la expansión celular, la permeabilidad celular (Hossain et al. 2010), la fotosíntesis, la producción de hojas, el crecimiento (Chapin 1980; Clarkson \& Hanson 1980; Evans 1972; Zhao et al. 2003, 2005), y el rendimiento, como sucedió con los tratamientos T6 y T12, en los que se obtuvieron bajos resultados, como se muestran en la tabla 5 de la prueba de Duncan $(\mathrm{p}<0.05)$.

Los resultados obtenidos en esta investigación indican que la aplicación foliar de biofertilizante de 15 L.ha ${ }^{-1}$, mejora significativamente el crecimiento y desarrollo de las plantas de Sacha inchi, demostrando la capacidad estimuladora del crecimiento. Estudios similares hechos por Basantes, (2010); Bejarano (2004) y Jiménez (2008), reportan que concentraciones del biol al 25\% $\left(50\right.$ L.ha $\left.^{-1}\right)$ logran obtener estos efectos en cultivos de brócoli (Brassica oleraceavar. Legacy) y en banana (Musa spp.).

A pesar de que este estudio no fue llevado hasta el periodo de cosecha, se observó un efecto positivo de los biofertilizantes durante la etapa vegetativa y reproductiva, esperándose resultados favorables al momento de la cosecha. La etapa vegetativa es considerada crítica para obtener niveles de producción óptimos (Álvarez, 2011).

Tabla 4. Significancias obtenidas en el análisis de variancia para los parámetros de crecimiento y desarrollo de P. volubilis $\mathrm{L}$

\begin{tabular}{|c|c|c|c|c|c|c|c|}
\hline Fuente de variabilidad & GI & C. Media & F. Valor & Pr. $>$ F & Sign. & $\mathbf{R}^{2}$ & Coef. Var. \\
\hline \multicolumn{8}{|l|}{ Altura de planta $(\mathrm{cm})$} \\
\hline Tutor & 1 & 24,17 & 6,90 & 0,0162 & * & $85,93 \%$ & $7,40 \%$ \\
\hline Biofertilizantes & 5 & 78,95 & 22,52 & $<, 0001$ & $* *$ & & \\
\hline Tutor * Biofertilizantes & 5 & 0,32 & 0,09 & 0,9925 & ns & & \\
\hline \multicolumn{8}{|l|}{ Días a la aparición de guía } \\
\hline Tutor & 1 & 1708,44 & 45,01 & $<, 0001$ & $* *$ & $78,91 \%$ & $11,78 \%$ \\
\hline Biofertilizantes & 5 & 161,31 & 4,25 & 0,0085 & $* *$ & & \\
\hline Tutor * Biofertilizantes & 5 & 56,98 & 1,50 & 0,234 & ns & & \\
\hline \multicolumn{8}{|l|}{ Días a la floración } \\
\hline Tutor & 1 & 186,78 & 20,35 & 0,0002 & $* *$ & $96,72 \%$ & $3,60 \%$ \\
\hline Biofertilizantes & 5 & 959,73 & 105,08 & $<, 0001$ & $* *$ & & \\
\hline Tutor * Biofertilizantes & 5 & 9,58 & 1,04 & 0,4198 & ns & & \\
\hline \multicolumn{8}{|l|}{ Número de flores / planta } \\
\hline Tutor & 1 & 584,03 & 43,84 & $<0,0001$ & $* *$ & $91,95 \%$ & $11,32 \%$ \\
\hline Biofertilizantes & 5 & 249,45 & 18,72 & $<0,0001$ & $* *$ & & \\
\hline Tutor * Biofertilizantes & 5 & 169,63 & 12,73 & $<0,0001$ & $* *$ & & \\
\hline \multicolumn{8}{|l|}{ Días a la frutificación } \\
\hline Tutor & 1 & 58,78 & 2,99 & 0,0992 & ns & $89,95 \%$ & $3,71 \%$ \\
\hline Biofertilizantes & 5 & 388,27 & 19,75 & $<0,0001$ & $* *$ & & \\
\hline Tutor * Biofertilizantes & 5 & 249,64 & 12,70 & 0,0001 & $* *$ & & \\
\hline \multicolumn{8}{|l|}{ Número de frutos / planta } \\
\hline Tutor & 1 & 4466,69 & 213,60 & $<0,0001$ & $* *$ & $95,73 \%$ & $10,64 \%$ \\
\hline Biofertilizantes & 5 & 624,82 & 29,88 & $<0,0001$ & ** & & \\
\hline Tutor * Biofertilizantes & 5 & 180,43 & 8,63 & 0,0002 & $* *$ & & \\
\hline
\end{tabular}


Tabla 5a. Efecto de la interacción tutor vivo y biofertilizantes en los parámetros de estudio de Plukenetia volubilis L. $(\mathrm{p}<0,05)$.

\begin{tabular}{|c|c|c|c|c|c|c|}
\hline \multirow[b]{2}{*}{ Parámetros de estudio } & \multicolumn{6}{|c|}{ Sistema Tutor Vivo (TV) } \\
\hline & $\begin{array}{c}\text { T1 } \\
\text { TV-BFTV }\end{array}$ & $\begin{array}{c}\text { T2 } \\
\text { TV-BHP }\end{array}$ & $\begin{array}{c}\text { T3 } \\
\text { TV-BHHL }\end{array}$ & $\begin{array}{c}\text { T4 } \\
\text { TV-BHC }\end{array}$ & $\begin{array}{c}\text { T5 } \\
\text { TV-B }\end{array}$ & $\begin{array}{c}\text { T6 } \\
\text { TV-SB }\end{array}$ \\
\hline Altura de planta a los 50 días (cm) & $27,7 \mathrm{ab}$ & $29,7 \mathrm{a}$ & $27,3 \mathrm{ab}$ & $26,6 \mathrm{ab}$ & $26,0 \mathrm{~b}$ & $19,5 c$ \\
\hline Días a la aparición de tallo guía & $55,0 a b$ & $54,7 \mathrm{ab}$ & $61,7 \mathrm{a}$ & $59,7 \mathrm{ab}$ & 62,0 a & $62,0 \mathrm{a}$ \\
\hline Días a la floración & 78,7 def & 82,0 cde & 82,0 cde & 78,7 def & $86,7 \mathrm{c}$ & $111,3 \mathrm{a}$ \\
\hline Número de flores / planta & 28,3 cde & $29,7 \mathrm{~cd}$ & $29,3 \mathrm{~cd}$ & $34,0 \mathrm{c}$ & 26,7 de & $26,3 \mathrm{de}$ \\
\hline Días a la fructificación & $123,0 \mathrm{C}$ & $119,7 \mathrm{~cd}$ & 116,3 cde & 115,3 de & $121,0 \mathrm{~cd}$ & $139,0 \mathrm{~b}$ \\
\hline Número de frutos / planta & $20,0 \mathrm{f}$ & 36,3 de & 38,7 de & 39,7 de & 37,3 de & $19,0 \mathrm{f}$ \\
\hline
\end{tabular}

${ }^{*}$ Medias con diferentes letras en una misma fila difieren estadísticamente según la Prueba de Duncan $(p<0,05)$.

Tabla 5b. Efecto de la interacción tutor espaldera y biofertilizantes en los parámetros de estudio de Plukenetia volubilis $\mathrm{L}$. $(\mathrm{p}<0,05)$.

\begin{tabular}{|c|c|c|c|c|c|c|}
\hline \multirow[b]{2}{*}{ Parámetros de estudio } & \multicolumn{6}{|c|}{ Sistema Espaldera (E) } \\
\hline & $\begin{array}{c}\text { T7 } \\
\text { E-BFTV }\end{array}$ & $\begin{array}{c}\text { T8 } \\
\text { TV-BHP }\end{array}$ & $\begin{array}{c}\text { T9 } \\
\text { E-BHHL }\end{array}$ & $\begin{array}{c}\text { T10 } \\
\text { E-BHC }\end{array}$ & $\begin{array}{l}\text { T11 } \\
\text { E-B }\end{array}$ & $\begin{array}{c}\text { T12 } \\
\text { E-SB }\end{array}$ \\
\hline Altura de planta a los 50 días $(\mathrm{cm})$ & $25,9 \mathrm{~b}$ & $27,9 a b$ & $26,3 a b$ & $25,3 b$ & $24,3 \mathrm{~b}$ & $17,2 \mathrm{c}$ \\
\hline Días a la aparición de tallo guía & $42,0 \mathrm{~cd}$ & $38,0 \mathrm{~d}$ & $42,0 \mathrm{~cd}$ & $42,0 \mathrm{~cd}$ & 48,7 bc & $59,7 a b$ \\
\hline Días a la floración & $76,7 \mathrm{f}$ & $74,3 \mathrm{f}$ & $74,3 f$ & $74,3 f$ & $83,7 \mathrm{~cd}$ & $108,7 \mathrm{~b}$ \\
\hline Número de flores / planta & 55,3 a & $42,7 \mathrm{~b}$ & $35,0 \mathrm{c}$ & $31,7 \mathrm{~cd}$ & 26,7 de & $26,3 \mathrm{de}$ \\
\hline Días a la fructificación & 113,7 de & $109,0 \mathrm{e}$ & 111,3 de & 111,3 de & $121,0 \mathrm{~cd}$ & $143,0 \mathrm{a}$ \\
\hline Número de frutos / planta & $56,7 \mathrm{c}$ & $61,0 \mathrm{~b}$ & 65,0 a & 66,0 a & $42,7 d$ & 33,3 e \\
\hline
\end{tabular}

${ }^{*}$ Medias con diferentes letras en una misma fila difieren estadísticamente según la Prueba de Duncan $(p<0,05)$. 


\section{CONCLUSIONES}

Los resultados indican que la aplicación de los biofertilizantes redujo el periodo vegetativo e incrementó el número de flores y frutos de Sacha inchi. Los tratamientos T4 y T8 redujeron los días a la fructificación; mientras que el mayor número de frutos por planta se logró con los tratamientos T4, T9 y T10, mejorando la productividad de plantas de $P$. volubilis $\mathrm{L}$.

\section{AGRADECIMIENTO}

A los Directivos e investigadores de la Asociación para la Conservación de la Cuenca Amazónica ACCA, por el financiamiento del trabajo de investigación en su programa de Becas y a los socios de la Asociación de Productores Agroecológicos de Sacha Inchi - Manu, liderados por el Sr. Martín Cruz Quispe, por el apoyo en las labores de campo.

\section{BIBLIOGRAFÍA}

Álvarez, R.A. 2011. Efecto de los Biofertilizantes Líquidos de Producción Local "Bioles", sobre el Desarrollo de Síntomas Causados por el Virus del Mosaico de la Calabaza (SqMV) en el Cultivo de Melón (Cucumis melo L.) var. Edisto en Condiciones de Invernadero. Tesis de Grado. Facultad de Ingeniería en Mecánica y Ciencias de la Producción. Escuela Superior Politécnica del Litoral. Guayaquil, Ecuador. 63 pp

Álvarez, J.; Vanegas, S.; Soto, C.; Chávez, A.; Zabala, L. 2011. Uso de fertilizantes químicos y orgánicos en cebolla (Allium cepa L.). México, MX. Revista de investigación y difusión c i entifica a gropecuaria. (http://www.ucol.mx/revaia/portal/pdf/2011/ma yo/3.pdf). Acceso: 01/05/2012

Alcívar, E., Párraga, Flor-María y Guzmán, A. 2012. Efecto del Biol enriquecido con bacterias Acidolácticas en la Productividad del Cultivo de Maní (Arachis hipogaea L.) ESPAM - MFL. 2011. Tesis de pre-grado. Escuela Superior Politécnica Agropecuaria de Manabí Manuel Félix López, Facultad de Ingeniería Agrícola, Calceta, Provincia de Manabí, Ecuador. 89 pp.

Arévalo, G. 1996. El Cultivo de Sacha Inchi (Plukenetia Volúbilis L.) en la Amazonía. Instituto de Investigación Agraria, Proyecto Suelos Tropicales. Lima, Perú. 68 p.

Basantes, D. 2010. “Elaboración y aplicación de dos tipos de Biol en el cultivo de brócoli (Brassica oleraceavar. legacy),". Tesis de Grado. Escuela Superior Politécnica de Chimborazo. p. 123. (http://dspace.espoch.edu.ec/handle/123456789 /352???help.index???). Acceso: 24/03/2013.

Bejarano, C.; Méndez, H. y Gordillo, E. 2004. Fertilización Orgánica Comparada con la Fertilización Química en el Cultivo de Fréjol (Phaseolus vulgaris), para minimizar el efecto de Degradación del Suelo. Tesis de pre-grado. Universidad Técnica del Norte. Facultad de Ingeniería en Ciencias Agropecuarias y Ambientales. Provincia de Ibarra, Ecuador. 128 pp.

Bonilla, I. 2000. Capítulo 8: Introducción a la nutrición mineral de las plantas. Los elementos minerales In: Azcón-Bieto, J. \& Talón, M. (Eds) Fundamentos de la Fisiología Vegetal. p. 83-97

Capulin-Grande, J.; Núñez-Escobar, R.; AguilarAcuña, J. L.; Estrada-Botello, M.; SánchezGarcía, P.; Mateo-Sánchez, J. J. 2007. Uso de estiércol líquido de bovino acidulado en la producción de pimiento morrón. Revista Chapingo Serie Horticultura 13(1): 5-11.

Castro P. 2007. Sacha inchi: situación actual del cultivo y oportunidades de mercado. Documento técnico Oficinal. Dirección de Promoción Agraria de San Martín. - Dirección Regional Agraria de San Martín, Perú. 35 pp.

Castillo, S.E.F.; Castillo, V.S.F. \& Reyes, A.C.E. 2010. Estudio fitoquímico de Pluckenetia volubilis L. y su efecto antioxidante en la lipoperoxidación inducida por Fe3/ascorbato en hígado de Rattus rattus var. Albinus. UCVScientia, 2: 11-21.

Castillo-Sánchez, L., Jiménez-Osornio, J., \& Delgado-Herrera, M. 2012. Actividad biológica in vitro del extracto de Capsicum chinense Jacq contra Bemisia tabaci Genn. Revista Chapingo. Serie horticultura, 18(3), 345-356. (http://www.scielo.org.mx/scielo.php?script=sc i_arttext\&pid=S1027-152X2012000300008\& $\overline{l n} \mathrm{~g}=$ es\&tlng=es.10.5154/r.rchsh.2011.04.016.). Acceso: 12/08/2013.

Chapin III F.S 1980. The mineral nutrition of wild plants. Annual Review of Ecology and Systematic 11, 233-260.

Clarkson D, Hanson T. 1980. The mineral nutrition of higher plants. Annual Review of Plant Physiology 31, 239-298.

Criollo, H., Lagos, T., Piarpuezan, E., y Pérez, R.. 2011. The effect of three liquid bio-fertilizers in the production of lettuce (Lactuca sativa L.) and cabbage (Brassica oleracea L. var. capitata). Agronomía Colombiana, 29(3), 415-421. (http://www.scielo.org.co/scielo.php?script=sci _arttext\&pid=S0120-99652011000300010\& 
lng=en\&tlng=en). Acceso: 01/08/2014.

Dávila S. 2008. Efectos de la rotación y de biol en la Producción orgánica de dos cultivares de espinaca (Spinacea oleracea L.). Tesis para optar el título de Ingeniero Agrónomo. Universidad Nacional Agraria La Molina, Lima, Perú. 89 pp.

De Marcano, A.; Vargas, N; Pire, A. 2005. Efecto de extractos vegetales y fungicidas sintéticos sobre el crecimiento micelial in vitro de Sclerotium rolfsii y Thielaviopsis basicola. Rev. Fac. Agron., Caracas, v. 22, n. 4. Disponible en (http://www.scielo.org.ve/scielo.php?script=sci arttext\&pid $=$ S0378-78182005000400001\& $\overline{l n} \mathrm{n}=\mathrm{es} \& n \mathrm{~mm}=\mathrm{iso})$. Acceso: $12 / 08 / 2014$.

Elorza, P., López, M., Hernández, A.D., Olmedo, G., Domínguez, C, y Maruri, J.M. 2007. Efecto del tipo de tutor sobre el contenido de vainillina y clorofila en vainas de vainilla (Vanilla planifolia Andrews) en Tuxpan, Veracruz, México. Revista Cientifica UDO Agrícola, 7 (1): 228-236.

Evans GC. 1972. The cuantitative análisis of plant growth. Studies in ecology. Vol 1 Blackwell Scientific Publication. London 45-68 pp.

Gomero L. y Velásquez A. 1999. Manejo Ecológico de Suelos: Conceptos, Experiencias y Técnicas. Primera Edición. Editado por la Red de Acción en Alternativas al Uso de Agroquímicos (RAAA). Lima, Perú. Pág. 170-190.

Gómez, A.K.; Gómez, A.A. 1984. Statistical Procedures for Agricultural Research. 2da. Ed. John Willey y Sons Inc. USA. 680p.

Gorriti, A.; Arroyo, J.; Quispe, F.; Cisneros, B.; Condorhuamán, M.; Almora, Y. \& Chumpitaz, V. 2010. Toxicidad oral a 60 días del aceite de sacha inchi (Pluckenetia volubilis L.) y linaza (Linum usitatissimun L.) y determinación de la dosis letal 50 en roedores. Revista Peruana de Medicina Experimental y Salud Pública, 27: 352-360.

Hossain D, Hanafi M, Talib J, Hamdan J. 2010. Effects of nitrogen, phosphorus and potassium levels on kenaf (Hibiscus cannabinus L.) Growth and photosynthesis under nutrient solution. Journal of Agricultural Science, 2: 4956.

Jiménez, M.L. 2008. "Effect of the nutritional status of banana (Musa spp.) on leaf disease infestation by Mycosphaerella fijiensis Morelet in Ecuador". Doctoral Thesis. Leuven (BEL).

Litterick, A., Wood, M. 2009. "Chapter 5. The use of composts and compost extracts in plant disease control," en Disease Control in Crops: Biological and Environmentally-Friendly
Approaches, Dale Walters, Ed. Edinburgh, UK Wiley-Blackwell, pp. 93-117.

Kovacik J, Klejdus B, Backor M, Repcak M (2007) Phenylalanine ammonia-lyase activity and phenolic compounds accumulation in nitrogendeficient Matricaria chamomilla leaf rosettes. Plant Science, 172: 393-399.

Manco, E. 2006. Cultivo de Sacha Inchi. Situación y Avances del cultivo de Sacha Inchi en el Perú. Dirección de Investigación Agraria. Instituto Nacional de Investigación y Extensión Agraria. Estación Experimental "El Porvenir, Tarapoto, San Martín, Perú. 11 pp.

Márquez-Dávila, K.; Cayotopa, T.J.; Arévalo, G.E.; Vivanco, N. U.; Arévalo, Q. J . 2007. Diagnóstico y niveles de inoculo del nematodo que afecta a la raíz de sacha inchi (Plukenetia volubilis L.) en el Perú. Fitopatología, 42 (2): 52.

Marschner H (1995) Mineral Nutrition of Higher Plants $\left(2^{\text {nd }}\right.$ Ed), Academic Press, London, 889 pp.

Mcbride, F. 1951. Euphorbiaceae. In Flora of Peru. Botanical Series vol. 13, Part IIIA. Field Museum of Natural History. pp. 115-118.

Merino, C.; Sotero, V.; Del Castillo, D.; Vásquez, G.; Cachique, D. \& Vásquez Ocmín, P. 2008. Caracterización química de nueve ecotipos de Plukenetia volubilis L. de los departamentos de Loreto y San Martin. Folia Amazónica, 17: 3945.

Parry MAJ, Flexas J, Medrano H. 2005. Prospects for crop production under drought: research priorities and future directions. Annals of Applied Biology, 147:211-226.

Peña, S.; Lara, I.; Zárate, P.; Lolay, M. \& Marín, P. 2008. Protocolo del cultivo de Sacha inchi. Informe final del Subproyecto: "Adaptación y validación participativa de paquete tecnológico para la producción competitiva del sacha Inchi (Plukenetia volubilis L.) en la Cuenca del Perené. Centro de Investigación, Educación y Desarrollo (CIED) Selva Central, Junín - Perú. $86 \mathrm{p}$.

Perdomo, R. y Hampton, H. 1970. Ciencia y tecnología del suelo. Centro de Producción de Materiales. Universidad de San Carlos de Guatemala. 336 p.

Rodríguez-Dimas, N.; Cano-Rios, P.; Favela Chávez, E.; Figueroa-Viramontes, U.; De PaulÁlvarez, V.; Palomo-Gil, A.; MárquezHernández, C.; Moreno-Reséndez, A. 2007. Vermicomposta como alternativa orgánica en la producción de tomate en invernadero. Revista Chapingo Serie Horticultura, 13 (2): 185-192. 
Sánchez, E.P. y Terán, G. 2009. Evaluación de la Fertilización Química y Orgánica en el Cultivo de Lechuga Variedad (Verpia) en la Comunidad de Florencia - Tabacundo, Provincia De Pichincha. Tesis de pre-grado. Universidad Técnica del Norte. Facultad de Ingeniería en Ciencias Agropecuarias y Ambientales. Provincia de Ibarra, Ecuador. $115 \mathrm{pp}$.

SAS Institute. 2004. Statistical Analysis System (Version 9). Copyright (c) by SAS Institute Inc., Cary, NC, USA.

Seguel S., O, García de C., Víctor, \& Casanova P., M. 2003. Variación en el Tiempo de las Propiedades Físicas de un Suelo con Adición de Enmiendas Orgánicas. Agricultura Técnica, 63 (3): 287297. http://www.scielo.cl/scielo.php?script= sci_arttext\&pid=S0365-28072003000300008\& $\operatorname{lng}=$ es\&tlng=es. $10.4067 /$ S0365-280720030 00300008. Aceso: 11/08/2014.

Soria F., M. J.; Ferrera-Cerrato, R.; Etchevers B., J.; Alcantar G., G.; Trinidad S., A.; Borges G., L.; Pereyda, P. 2001. Producción de biofertilizante mediante biodigestión de excreta líquida de cerdo. Revista Terra Latinoamericanak, 19 (4): 353-362.

Xiang-wen P, Wen-bin L, Qiu-ying Z, Yan-hua L, Ming-shan L. 2008. Assessment on Phosphorus Efficiency Characteristics of Soybean Genotypes in Phosphorus-Deficient Soils. Agricultural Sciences in China, 7: 958-969.

Zhao D, Reddy KR, Kakani VG, Read JJ, Carter GA. 2003. Corn (Zea mays L.) growth leaf pigment concentration, photosynthesis and leaf hyperspectral reflectance properties as affected my nitrogen supply. Plant Soil, 257: 205-217.

Zhao D, Reddy KR, Kakani VG, Read JJ, Koti S. 2005. Selection of optimum reflectance ratios for estimating leaf nitrogen and chlorophyll concentrations of field-grown cotton. Agronomy Journal, 97: 89-98.

Recibido: 13/05/2014

Aceptado para publicación: 15/07/2014 
